\title{
1 Flow past a plate in the vicinity of a free surface
}

I-H. Liu, J. Riglin, W. Schleicher and A. Oztekin ${ }^{1)}$

Mechanical Engineering and Mechanics, P. C. Rossin School of Engineering and Applied Science, Lehigh University, Bethlehem, Pennsylvania 18015

\section{Abstract}

Two-dimensional transient simulationsare performed to investigate characteristics of flow past a plate normal to a stream.Free surface effects on the flow dynamics is the primary focus of this study. Varying plate depths are simulated to examine the variation of force coefficients and vortex shedding patterns. The $k-\omega$ Shear Stress Transport $(k-\omega \mathrm{SST})$ turbulence model and Volume of Fluid(VOF)multiphase model are employed to predict characteristics of freesurface flow.

11 Flow past the plates are simulated at distances of $0.75 \mathrm{~m}, 0.06 \mathrm{~m}, 0.05 \mathrm{~m}, 0.045 \mathrm{~m}$, and $0.03 \mathrm{~m}$

12 below the free surface with corresponding local Froude numbers $(F r)$ of $0.18,0.65,0.71,0.75$, 13 and 0.92 .As the plate gets closer to the surface the drag coefficient decreases from $3.86(F r=0.18)$ 14 to $2.18(F r=0.92)$ and the Strouhal number increases from $0.125(F r=0.18)$ to $0.355(F r=0.92)$. 15 A jet-like flow formed from the surface is observed on top of the plate. Vortices from the top 16 surface of the plate dissipate into smaller eddies due to the free surface presence, resulting in 17 asymmetric vortex shedding downstream. Flows presented here are beneficial for designing and 18 optimizing systems that harvest energy from marine currents.

\section{Keywords}

20 Marine energy; Free surface; Computational Fluid Mechanics; Drag coefficient

\footnotetext{
${ }^{1)}$ Author to whom correspondence should be addressed. Electronic mail: alo2@lehigh.eduPhone: +1-610-758-4343
} 


\section{Introduction}

Computationalfluid dynamics (CFD) is a powerful tool for solving flow problems. Proper

23 numerical simulationsare a valuable proxy for studyingfluid flow characteristicswhile

24 minimizing costly experiments. Understanding the flow around a rectangular bluff body is of

25 practical importance in various fields of engineering. Structures such as bridge decks, platforms,

26 offshore pipelines, andhydropower systemsoften interact with the freesurface.The normal

27 rectangular prism is a simple geometry used to understand complex phenomena such as flow

28 separation, wake instabilities, the unsteady vortex shedding formation, and the force acting on

29 the cylinder. Estimating structural hydrodynamic forces is an important design consideration for

30 offshore structures under unsteady flow conditions. Hydrokinetic turbines for river current

31 applications typically operate near surfaces and the effect of the free surface on the performance

32 of these energy harvesting devices could be profound.

33 Hydrokinetic power is an alternative clean energy source to conventional power such as

34 fossil fuels and nuclear power. Hydrokinetic turbines have been studied by various research

35 groups;however, theseengineering designs are limited by water depth. In general, the blades are

36 placed perpendicular to the flow path in order to extract the energy from rivers. A single flat

37 plate submerged at different water depthsisinvestigated as a preliminary study for marine current

38 systems, where thepower extracted is directly proportional to the drag force exerted on the

39 blades.Maximizingthe drag force is therefore equivalent to maximizing the power extracted. The

40 free surface effectisstudied to determine a proper plate depth so sufficient power can be

41 generated while the hydropower system is operatednear the free surface.

42 Free surface flows are challenging hydraulic engineering scenarios. For instance, when a

43 cylinder is placed closer to the free surface, the forces acting on the cylinder become 
44 increasinglycomplicateddue to the interaction between the free surface and the cylinder. Also,

45 the wake behind structures and water surface deformation are altered in such free surface

46 scenarios as well. Several researchers approached this problem numerically (Miyata et al., 1990

47 andArslan et al., 2013) and experimentally (Miyata et al., 1990; Sheridan et al., 1997;Reichl et

48 al., 2005; Malavasi and Guadagnini, 2007;Negri et al., 2010; and Arslan et al., 2013) by

49 employing circular or rectangular cylinders.

Generally, hydrodynamic forces acting on the object decreasedwhen the object is closer

51 to the surface, as described in Miyata et al., (1990) and Malavasi and Guadagnini, (2007).

52 Asimultaneouslyoccurringabrupt drop in drag coefficient and increase in Strouhal number is

53 reported byMiyata et al., (1990)when the depth-radius ratio was down to 1.7.Malavasi and

54 Guadagnini, (2007)experimentally investigated a rectangular cylinder submerged in a water

55 channel at various depths. They found that the drag coefficient decreases drastically and Strouhal

56 number increases with decreasing depth of the cylinder.Both Miyata et al., (1990) and Sheridan

57 et al., (1997) studied the wake structure of flow past a circular cylinder close to the free surface.

58 The presence of the cylinder, whichcaused the formation of surface waves, altered the dynamics

59 of the free surface and produceda jet-like flow near the free surface. When the cylinder was

60 shallowly submerged, the free surface distortion and asymmetric vortex shedding from the

61 cylinder occurred.Such asymmetric vortex shedding resulted in intenseturbulent fluctuations due

62 to theflow confinement on top.Negri et al., (2010)applied a self-synchronized phase averaging

63 technique to PIV velocity fields to study flow past a rectangular cylinder near a free surface. The

64 vortex shedding process and the wake evolution were reported by employing the phase-averaged

65 flow fields. Another study on the free surface deformation and wake behavior with respect to

66 Froude number is presented in Reichl et al., (2005). Results of two-dimensional 
67 simulationsshowed that the surface deformation was sensitive to the Froude number.The surface

68 deformation and the intensity of surface wavesbecame substantial as the Froude number

69 increased from 0.35 to 0.40 .Arslan et al., (2013) investigated the flow field of partially

70 submerged rectangular cylinder by conducting large eddy simulations and experimental

71 measurements. They concluded that the magnitude of the drag force is very sensitive to the

72 submergence ratio.

73 Flow pastbluff bodies in an infinite fluid domain has been widely studied both

74 experimentally and numerically.Simple geometries such as circular cylinders, rectangular prisms,

75 and flat plates have been investigated to understand the vortex dynamics and the drag and lift

76 forces.Fage and Johansen, (1927) experimentally measured the upstream and downstream

77 pressures for an inclined flat plate with various angle of attack.Knisely, (1990) experimentally

78 studied the Strouhal numbers for rectangular prisms with various aspect ratios at different angles

79 of attack. Both Narasimhamurthy and Andersson, (2009)and Najjar and Vanka,

80 (1995)numerically studied the structure and dynamics of the wake flow behind the flat plate.

81 Simulations were carried out for low Reynolds number andthe mean drag and pressure

82 coefficients were reported as well. In a recent work,Tian et al., (2013)investigated the effect of

83 aspect ratio for two-dimensional flow around rectangular prisms by conducting numerical

84 simulationsusing the $k$ - $\omega$ SST turbulence model. Bayraktar et al., (2014)conducted simulations

85 for flow past circular, square, and diamond cylinders in the proximity of no-slip wall. Results

86 revealed that the flow pattern loses periodicity as the gap between the cylinder and the wall

87 becomes smaller.Bayrakteretal., (2014) also documented that the mean value of drag coefficient 88 decreased substantially as the cylinder is placed near the wall. 
In spite of extensive studies on flow past bluff bodies, theunderstanding of the free

90 surface effectis still limited when a near-surface object is interacting with the free surface.

91 Investigating the effect of the free surface on the flow dynamicsis the primary objective of this

92 manuscript. The presence of the free surface affects both force coefficients and the wake

93 structure behind the object.In the present study, two-dimensional numerical simulations are

94 carried out to investigate the change of flow field and hydrodynamic forces on the normal plate

95 asthe plate depth is varied. A three-dimensional validation simulation was conducted to compare

96 with the experimental results provided by Malavasi and Guadagnini, (2007). The flow pattern,

97 drag and lift coefficients, and the Strouhal number as a function of plate depth (Froude number)

98 are reported in this paper. The present study willaid the design of a hydropower system for

99 riverand ocean current applications.

\begin{tabular}{|c|c|c|c|}
\hline \multicolumn{4}{|c|}{ NOMENCLATURE } \\
\hline$A$ & cross sectional area & $\Delta t$ & time step size \\
\hline$a, a_{1}$ & closure coefficient & $u_{i}, u_{j}$ & velocity in tensor notation \\
\hline$B$ & channel width & $u_{*}$ & friction velocity at the nearest wall \\
\hline$C$ & courant number & $U_{\infty}$ & water velocity \\
\hline$C_{D}$ & drag coefficient & $U_{\text {air }}$ & air velocity \\
\hline$C_{L}$ & lift coefficient & $U_{f}$ & $\begin{array}{l}\text { volume flux through the face based } \\
\text { on normal velocity }\end{array}$ \\
\hline$d$ & plate depth & $V$ & volume of cell \\
\hline$d^{*}$ & dimensionless plate depth & $\forall_{\text {air }}$ & volume of air within the cell \\
\hline$f$ & vortex shedding frequency & $\forall_{\text {cell }}$ & volume of a cell \\
\hline$F_{D}$ & drag force & $\forall_{\text {water }}$ & volume of water within the cell \\
\hline$F_{L}$ & lift force & $\mathbf{V}$ & upstream water velocity \\
\hline$F r$ & Froude number & $w$ & plate width \\
\hline$F_{1,} F_{2}$ & blending functions & $x_{i}$ & position vector in tensor notation \\
\hline & gravity & $\Delta x$ & grid width \\
\hline GCI & grid convergence index & $y$ & distance to the nearest wall \\
\hline$h$ & water depth & $y^{+}$ & dimensionless wall distance \\
\hline$h_{b}$ & $\begin{array}{l}\text { cylinder elevation above channel } \\
\text { floor }\end{array}$ & \multicolumn{2}{|c|}{ GREEK SYMBOLS } \\
\hline$h^{*}$ & $\left(h-h_{b}\right) / s$,non-dimensional depth & $\alpha$ & volume fraction \\
\hline$k$ & turbulent kinetic energy & $\beta, \beta^{*}$ & closure coefficient \\
\hline$l$ & cylinder cross-section length & $\delta_{i j}$ & Kroneckerdelta \\
\hline$L$ & plate height & $\mu$ & dynamic viscosity \\
\hline
\end{tabular}




\begin{tabular}{|llll|}
\hline$\dot{m}_{12}, \dot{m}_{21}$ & mass transfer between phase 1 and 2 & $v$ & kinematic viscosity \\
$N_{1}, N_{2}, N_{3}$ & number of cells & $v_{T}$ & eddy viscosity \\
$P$ & pressure & $\rho$ & density \\
$R e$ & Reynolds number & $\sigma_{k}, \sigma_{\omega}, \sigma_{\omega 2}$ & closure coefficients \\
$S$ & cylinder cross-section width & $\tau_{i j}$ & stress tensor \\
$S$ & mean rate-of-strain tensor & $\omega$ & specific dissipation rate \\
$t$ & time & $\Omega$ & vorticity magnitude \\
\hline
\end{tabular}

\section{Mathematical Model}

The two-dimensional transient simulation is regarded as an infinitely long prism

103 immersed in the flow. The basic flow physics and the hydrodynamic forces exerted on the

104 submerged object are investigated by conducting suchsimulations. The VOF method models two

105 immiscible fluids by solving mass and momentum conservation equations. The volume fraction

106 for each fluid in cells can be tracked throughout the domain. The airvolume fraction is defined by

107 a scalar function $\alpha$, which is the volume of air divided by the volume of the local cell.Volume

108 fraction is bounded between $0 \leq \alpha \leq 1$, corresponding to water-filled cells and air-filled cells,

109 respectively. When $0<\alpha<1$, the cell contains the interface between air and water. $\alpha$ is expressed

110 as

$111 \alpha=\frac{\forall_{\text {air }}}{\forall_{\text {cell }}}=\frac{\forall_{\text {air }}}{\forall_{\text {air }}+\forall_{\text {water }}}$

112 where $\forall_{\text {cell }}$ is the volume of a cell, $\forall_{\text {air }}$ is the volume of air within the cell, and $\forall_{\text {water }}$ is the

113 volume of the water within the cell. The mass and momentum equations for multiphase floware

114 writtenas ( seeHirt and Nichols, 1981 and Monaghan, 1994)

$115 \frac{\partial u_{i}}{\partial x_{i}}=\mathbf{0}$

$116 \frac{\partial}{\partial t}\left(\rho u_{i}\right)+\frac{\partial}{\partial x_{j}}\left(\rho u_{j} u_{i}\right)=-\frac{\partial P}{\partial x_{i}}+\frac{\partial}{\partial x_{j}}\left[\mu\left(\frac{\partial u_{j}}{\partial x_{i}}+\frac{\partial u_{i}}{\partial x_{j}}\right)\right]+\rho g_{i}$ 
117 where $u_{i}$ is the velocity, $\rho$ is the density, $\mu$ is the dynamic viscosity, $P$ is the pressure, and $g$ is

118 gravity. The density of the mixture is defined in terms of $\alpha$ as

$119 \rho=\alpha \rho_{1}+(1-\alpha) \rho_{2}$

120 where $\rho_{1}$ denotes the density of the primary phase (air) and $\rho_{2}$ denotes the density of the

121 secondary phase (water).Interface tracking between water and air is accomplished by solving the

122 continuity equation for the phase's volume fraction, which has the following form:

$123 \frac{\partial}{\partial t}\left(\alpha \rho_{1}\right)+\frac{\partial}{\partial x_{i}}\left(\alpha \rho_{1} u_{1}\right)=\dot{m}_{21}-\dot{m}_{12}$

124 where $u_{1}$ represents the velocity of the primaryphase and $\dot{m}_{21}$ and $\dot{m}_{12}$ are the mass transfer

125 between air and water.The plate is set to be perpendicular to the streamwise direction. The

126 upstream water speed, $U_{\infty}$, is the relative fluid speed with respect to the plate.

127 In order to find out how much energy can be extracted, the force acting on the plate must

128 be determined. The drag and lift coefficients $\left(C_{D}\right.$ and $\left.C_{L}\right)$ are defined as

$C_{D}=\frac{F_{D}}{\frac{1}{2} \rho U_{\infty}^{2} A}, C_{L}=\frac{F_{L}}{\frac{1}{2} \rho U_{\infty}^{2} A}$

130 where $F_{D}$ is the drag force, $F_{L}$ is the lift force, and $A$ is the area of the immersed object projected 131 over the incoming flow.

132 The Reynolds number is based on the upstream water velocity $U_{\infty}$ and the plate height $L$.

133 It is calculated as $R e_{2}=\rho_{2} U_{\infty} L / \mu_{2}$ where $\mu_{2}$ is the water dynamic viscosity. Strouhal number, $134 S t=f L / U_{\infty}$, is the dimensionless number utilized in the flow characterization of oscillating 135 flow mechanics, where $f$ is the vortexshedding frequency.

Open channel flow, such as rivers and spillways, involve the existence of a free surface

137 between air and water. In these cases, the free surface behavior becomes important. The flow is 
138 governed by the forces of gravity and inertia and it can be characterized by the dimensionless

139 parameter, Froude Number, $F r=U_{\infty} / \sqrt{g d}$ where $d$ is the distance between free surface and the

140 plate tip. The Froude number based on $d$ basically represents the local Froude number and is

141 suitable to characterize the effect of the free surface on the physics of flows near the submerged

142 object (Sheridan et al., 1997).

143 The schematic of the flow geometry is shown inFig. 1.Two-dimensional URANS VOF

144 simulations are conducted at $R e=50,000$. The entire fluid domain is divided into two parts:

145 water at the bottom and air on the top. The upstream water velocityis set to $U_{\infty}=0.5 \mathrm{~m} / \mathrm{sand}$ the air

146 velocityis set to $U_{\text {air }}=0.01 \mathrm{~m} / \mathrm{s}$. The plate heightis $L$. The plateis placed perpendicular to the

147 upstream flow inside a flow channel, whichis a simple duct with a height of $16 L$. The channel is

148 large enough to eliminatethe boundary effects from side walls. The coordinate origin is located at

149 the plate center. The inlet is $20 L$ upstream the plate to assure fully developed flow and the outlet

150 is $20 L$ downstream the plateto obtain wake dynamics free of outlet effects. The depth to length

151 ratio is $d^{*}=d / L$. Simulations are conducted for $d^{*}$ of $7.5,0.6,0.5,0.45$, and 0.3 . The

152 corresponding local Froude number calculated based on depths of plate are $0.18,0.65,0.71,0.75$,

153 and 0.92 . The property ratio between air and water for all the simulations presented in this

154 manuscript are described as $\rho_{1} / \rho_{2}=1.23 \times 10^{-3}, U_{\text {air }} / U_{\infty}=2.00 \times 10^{-2}, \mu_{1} / \mu_{2}=1.82 \times$

$15510^{-2}$. Modeling the flow past a plate normal to oncoming flow is achieved using ANSYS 14.5

156 Fluent simulation tool (see ANSYS theory guide and ANSYS user guide). Table 1 listed all the 157 parameter values used in the simulations. 


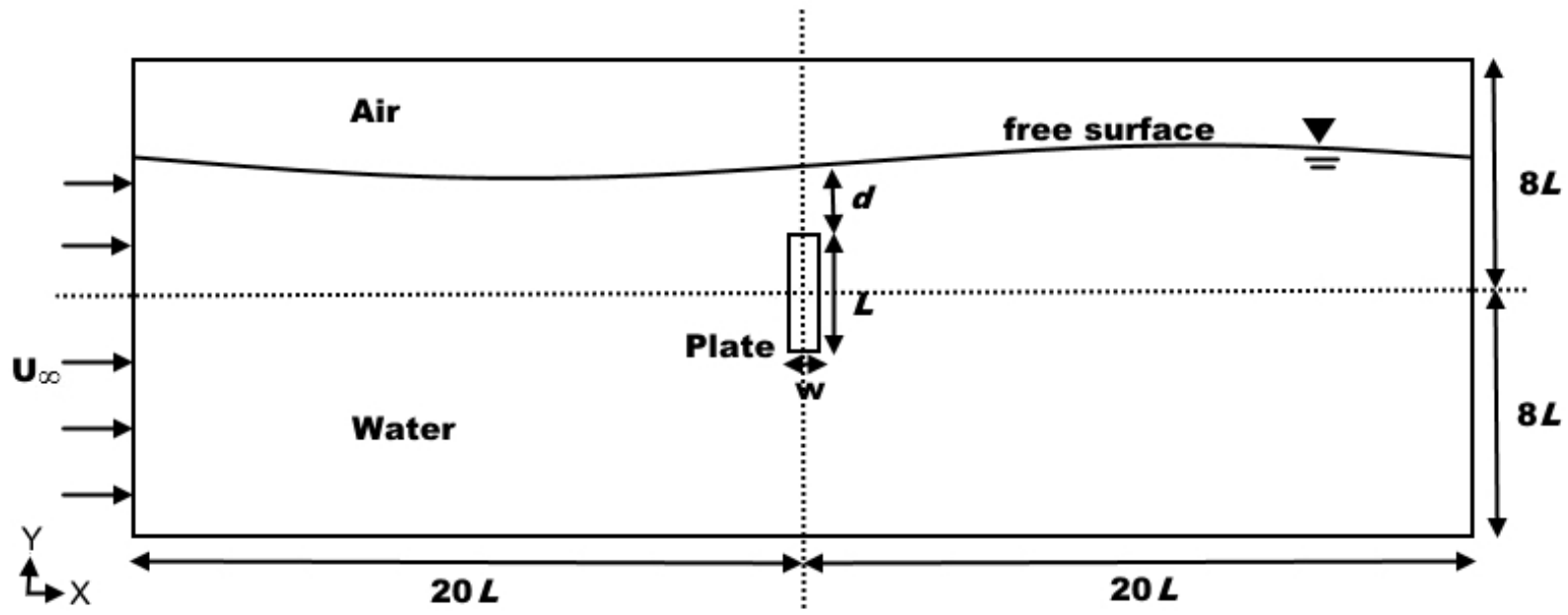

Fig.1. The schematic of the flow geometry.

161 Table 1. Parameter values used in the simulations

\begin{tabular}{|lrllll|}
\hline \multicolumn{4}{l}{ Parameter Values } \\
$A$ & 0.1 & {$\left[\mathrm{~m}^{2}\right]$} & $U_{\text {air }}$ & 0.01 & {$\left[\mathrm{~m} \mathrm{~s}^{-1}\right]$} \\
$g$ & 9.81 & {$\left[\mathrm{~m} \mathrm{~s}^{-2}\right]$} & $U_{\infty}$ & 0.5 & {$\left[\mathrm{~m} \mathrm{~s}^{-1}\right]$} \\
$L$ & 0.1 & {$[\mathrm{~m}]$} & $w$ & 0.01 & {$\left[\mathrm{~m}^{-1}\right.$} \\
$d$ & $0.75,0.06,0.03$ & {$[\mathrm{~m}]$} & $\rho_{2}$ & 1000 & {$\left[\mathrm{~kg} \mathrm{~m}^{-3}\right]$} \\
$\operatorname{Re}_{2}$ & 50,000 & {$[-]$} & $\mu_{2}$ & 0.001 & {$\left[\mathrm{~kg} \mathrm{~m}^{-1} \mathrm{~s}^{-1}\right]$} \\
\hline
\end{tabular}

The combination of two separate models is required when modeling flows of two fluids

164 separated by a free surface. Turbulence is modeled with the $k-\omega$ SST model (Menter, 1994) and

165 the VOF multiphase model (Hirt and Nichols, 1981 andMonaghan, 1994) is used to determine

166 interface dynamics separatingwaterfrom air. In order to examine the free surface effect, the VOF

167 model is necessary to track wherethe air-water interface is located. The $k$ - $\omega$ model is an effective

168 turbulence modelin predicting flow separation. The two equation $k$ - $\omega$ model solves for the

169 turbulence kinetic energy and the specific dissipation rate. The $k$ - $\omega$ SST model has been

170 formulated to avoid the upstream sensitivity of the standard $k-\omega$ model by combining the 
171 advantages of the $k-\omega$ model and $k$-emodel. The equations for turbulent kinetic energy and

172 specific dissipation rate are written in the followingform:(Menter, 1993,1994; and Wilcox, 2006)

$173 \quad \frac{\partial k}{\partial t}+u_{j} \frac{\partial k}{\partial x_{j}}=\tau_{i j} \frac{\partial u_{i}}{\partial x_{j}}-\beta^{*} k \omega+\frac{\partial}{\partial x_{j}}\left[\left(v+\sigma_{k} v_{T}\right) \frac{\partial k}{\partial x_{j}}\right]$

$174 \frac{\partial \omega}{\partial t}+u_{j} \frac{\partial \omega}{\partial x_{j}}=a S^{2}-\beta \omega^{2}+\frac{\partial}{\partial x_{j}}\left[\left(v+\sigma_{\omega} v_{T}\right) \frac{\partial \omega}{\partial x_{j}}\right]+2\left(1-F_{1}\right) \sigma_{\omega 2} \frac{1}{\omega} \frac{\partial k}{\partial x_{i}} \frac{\partial \omega}{\partial x_{i}}$

175 The eddy viscosity and the extra stress tensor are defined as

$176 \quad v_{T}=\frac{a_{1} k}{\max \left(a_{1} \omega, \Omega F_{2}\right)}$

$177 \quad \tau_{i j}=v_{T}\left(\frac{\partial u_{i}}{\partial x_{j}}+\frac{\partial u_{j}}{\partial x_{i}}-\frac{2}{3} \frac{\partial u_{k}}{\partial x_{k}} \delta_{i j}\right)-\frac{2}{3} k \delta_{i j}$

178 Here, $\Omega$ is the vorticity magnitude, $a, a_{1} \beta, \beta^{*}, \sigma_{k}, \sigma_{\omega}, \sigma_{\omega_{2}}$ are closure coefficients, $v$ is the

179 kinematic viscosity, $k$ is the turbulent kinetic energy, $\omega$ is the specific dissipation rate, $u_{j}$ is the

180 velocity, $S$ is the mean rate-of-strain tensor, and $F_{1}, F_{2}$ are the blending functions. Details of the

181 closure parameters can be found in Menter, (1994). Recently, this turbulence model is

182 successfully employed by the present authors to characterize turbulent flow structures near

183 micro-hydroturbines (Schleicher et al., 2013,2014a, 2014b,2014c).

The VOF model can depict two or more fluids by solving a single set of momentum

185 equations and tracking the volume fraction of each fluid throughout the domain. The implicit

186 scheme is used for time discretization for obtaining the face fluxes for all cells:

$\frac{\alpha_{1}^{n+1} \rho_{1}^{n+1}-\alpha_{1}^{n} \rho_{1}^{n}}{\Delta t} \forall_{c e l l}+\sum_{f}\left(\rho_{1}^{n+1} U_{f}^{n+1} \alpha_{1, f}^{n+1}\right)=\left(\dot{m}_{21}-\dot{m}_{12}\right) \forall_{c e l l}$ 
188 wheren +1 denotes the index for current time step while $n$ denotes the index for previous time 189 step. $\alpha_{1, f}$ is the face value of the airvolume fraction, and $U_{f}$ is the volume flux through the face 190 based on normal velocity.

The boundary conditions for the simulations are as follows. The mass flow rates for both

192 water and air are specified at the inlet. The computational domain behind the plateis large 193 enough so zero gauge pressureis imposed at the outlet.At the inlet and the outlet, the free surface 194 levelsare defined in the VOF model based on the plate depth. Ano-slip boundary condition is 195 invoked on the plate surfaces and the bottom boundary. Furthermore, ano shear boundary 196 condition is applied at the top boundary of the domain.

The Semi-Implicit Method for Pressure-Linked Equations (SIMPLE) solution method

198 was used to resolve the coupling between the pressure and the velocity fields. Discretization of 199 time, momentum, turbulent kinetic energy and specific dissipation rate were accomplished 200 through a second-order upwind scheme. The modified High Resolution Interface Capturing 201 (HRIC) discretization schemewas used to solve the volume fraction equation. The transient 202 simulation was stopped after the solution reached periodic stability.

\section{3. Validation}

In order to verify the accuracy of the multiphase simulation, a validation test is conducted. Malavasi and Guadagnini, (2007) provides experimental data of a rectangular

206 cylinder in the free surface flow. The transient three-dimensional validation simulation is to 207 simulate one of the cases presented in the reference. The schematic of the geometry is shown 208 inFig. 2. The channel is $5 \mathrm{~m}$ long with a cross-section width $B=0.5 \mathrm{~m}$. The rectangular cylinder is 209 fully submerged in water and the depth is represented as $h^{*}$, where $h^{*}=\left(h-h_{b}\right) / s$. Values of 
210 parameters used in the simulation are listed in Table 2. The case with the Reynolds number of

$2112.02 \times 10^{4}, h^{*}=4$, and the ratio $h_{b} / s=2.33$ is chosen to be modeled.

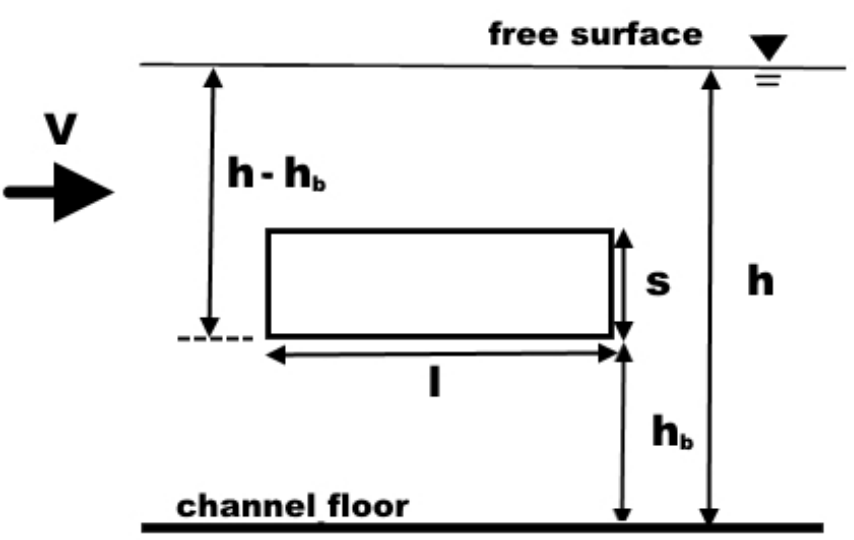

longitudinal view

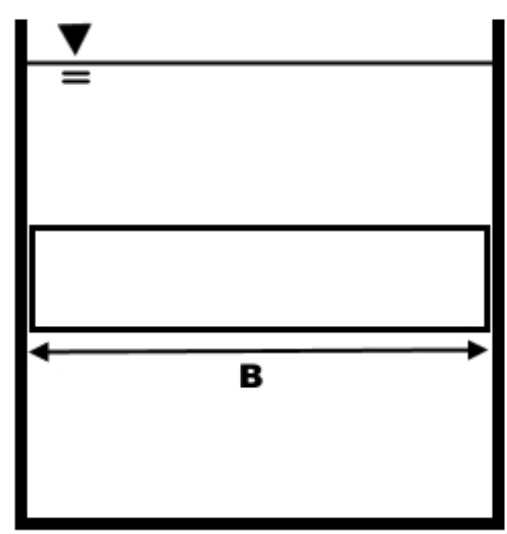

transverse view
212

213

214 Table 2. Parameter values used in the validation simulation
215

217 drag andthelift coefficient, and the Strouhal number observed from validation simulationare 1.69,

$218-0.67$, and 0.17 , respectively. These values are determined from the time signature of the drag

219 and the lift coefficient at flow time $24 \mathrm{~s}$ to $32 \mathrm{~s}$ in order to avoid the initial transient

220 effects.Predicted results from the present studyhave relative errors of less than $6 \%$ when

221 compared with the drag and the lift coefficients and the Strouhal number documented

222 byMalavasi and Guadagnini, (2007).Fig. 4depictsan instantaneous velocity and vorticity contours 
223 for flow time $30.3 \mathrm{~s}$. The black horizontal line above the rectangular cylinder indicates the free

224 surface location, which is slightly perturbed from its resting state.
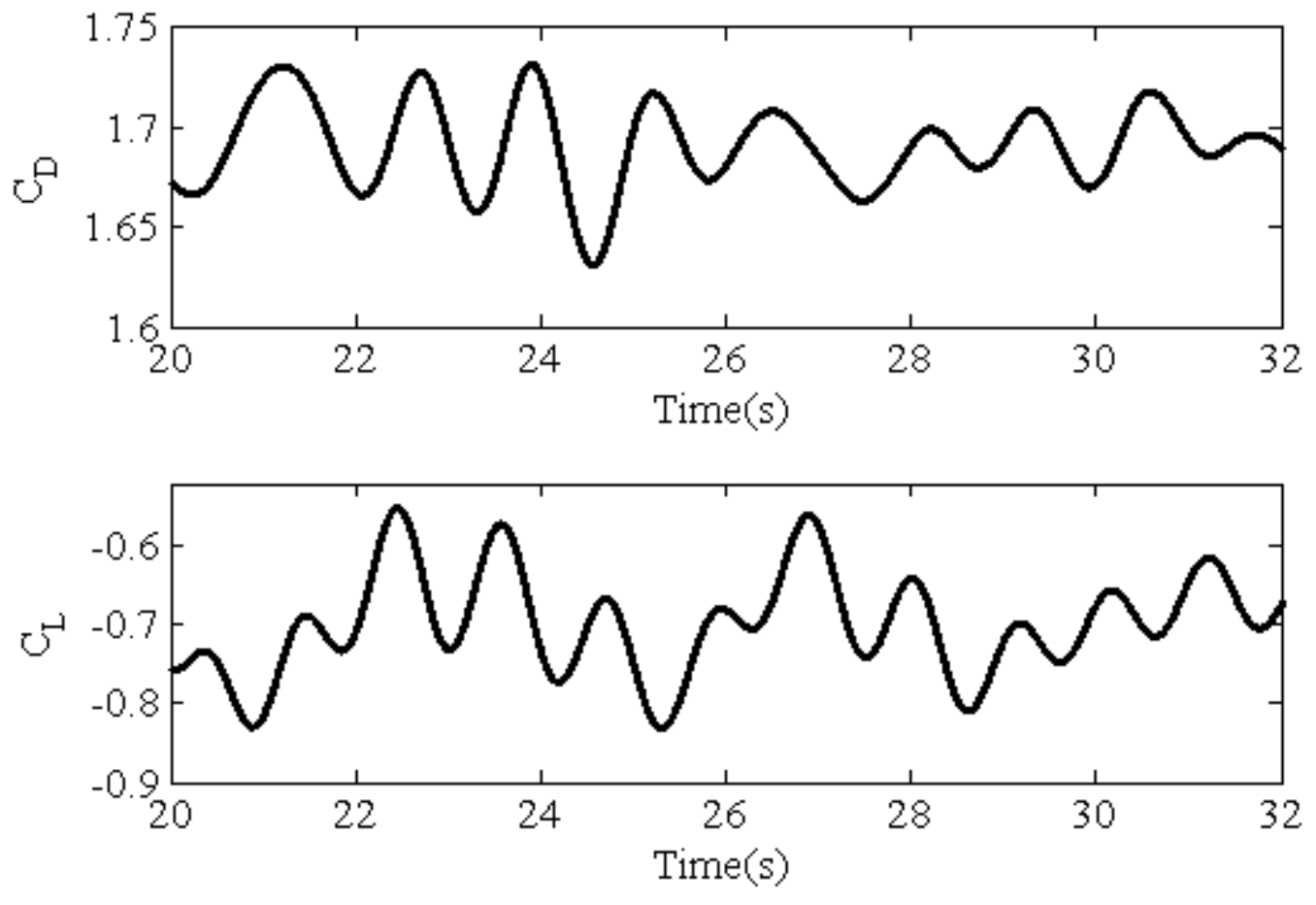

225

226 Fig.3. Drag and lift coefficients as functions of time. The validation simulation is conducted at $227 \quad \operatorname{Re}=2.02 \times 10^{4}, h^{*}=4$, and $h_{b} / s=2.33$. 


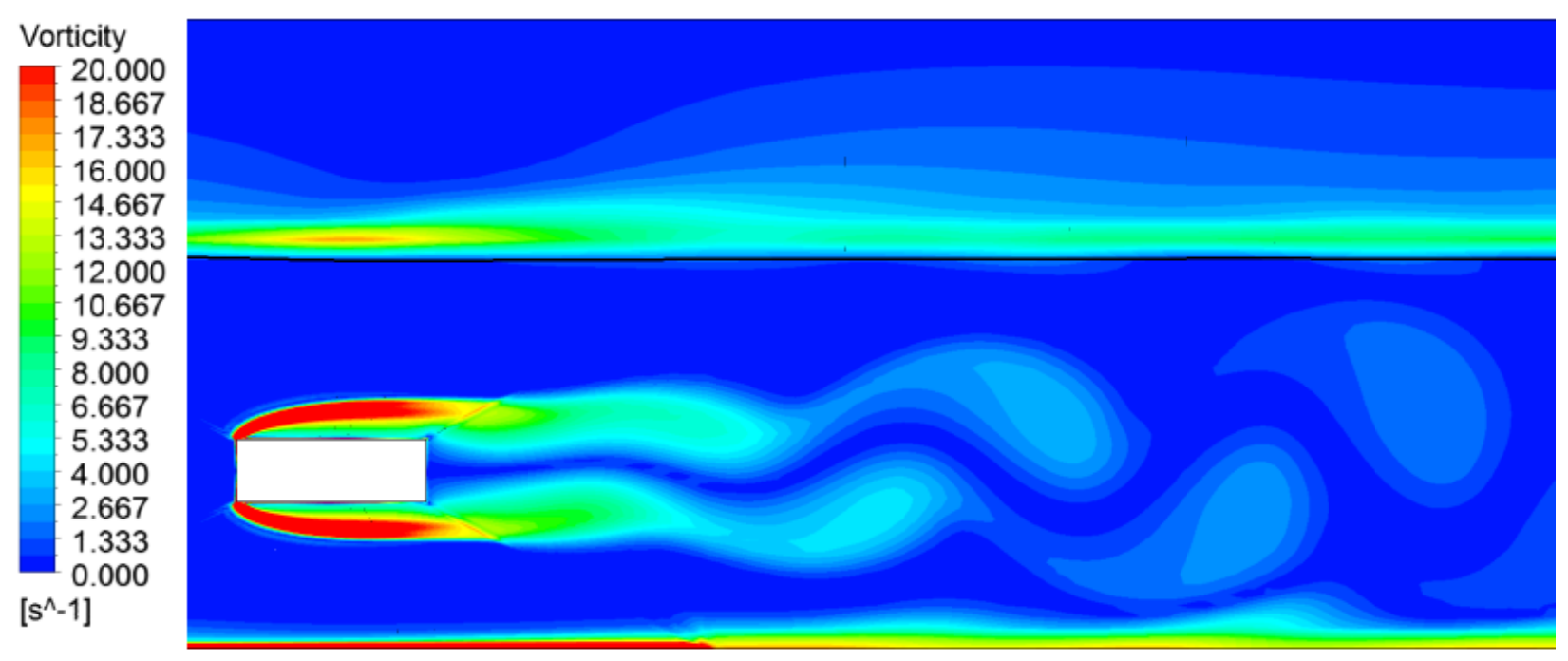

(a)

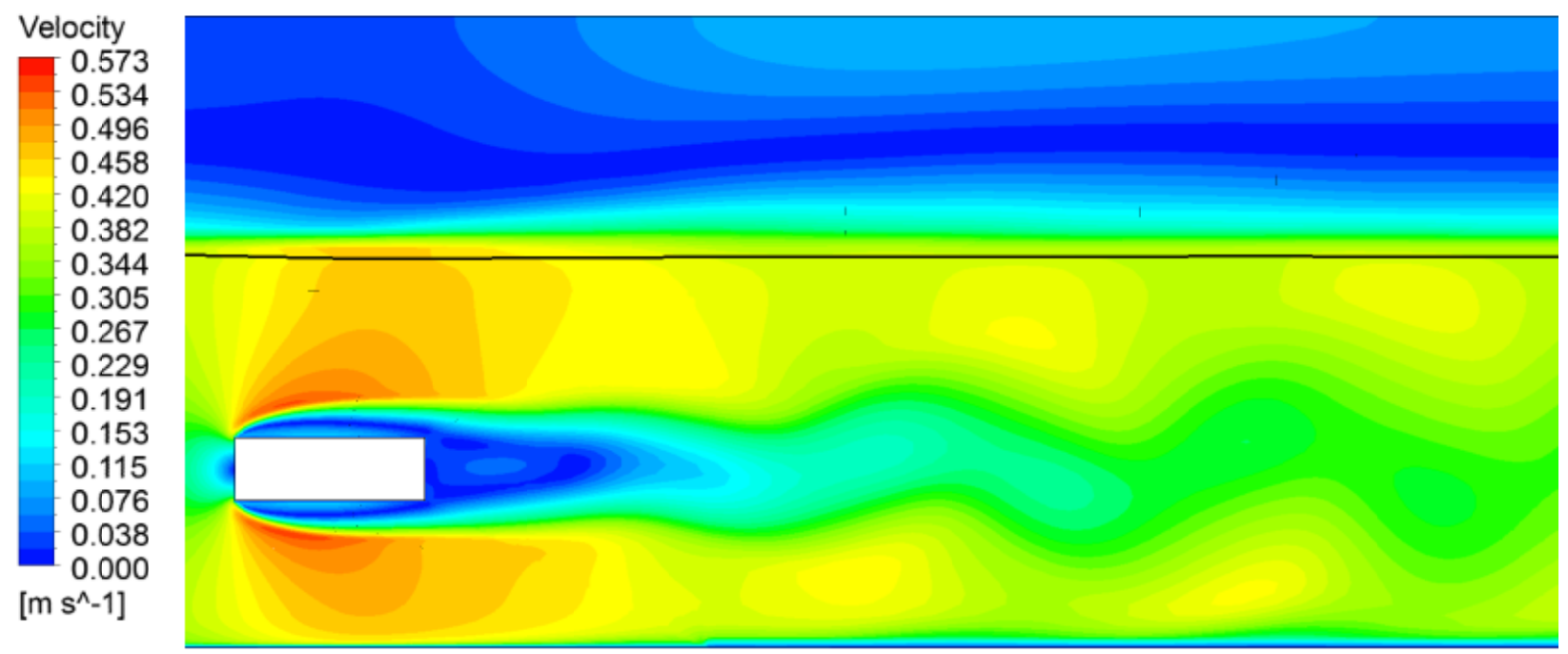

(b)

236 Fig.4. Instantaneous (a) vorticity and (b) velocity contours at $\mathrm{t}=30.3 \mathrm{~s}$. Validation simulation is 237 conducted at $\operatorname{Re}=2.02 \times 10^{4}, h^{*}=4$, and $h_{b} / s=2.33$.

\section{4. Mesh Optimization and Temporal Convergence}

The VOF model is susceptible to instabilities if non-orthogonal elements exist in the

240 discretized fluid mesh. Therefore, the mesh used for the multiphase simulations must be 
235 carefully chosen to allow for acceptable accuracy as well as numerical stability.Increasing the

236 number of cells in the mesh may produce more accurate simulation results; however, a higher

237 mesh density results in a substantially longer computational time and increased CPU usage.

A spatial convergence test is conducted in order to assess the numerical accuracy of the

239 simulations. The grid size used in this paper is verified usingthe Richardson extrapolation based

240 Grid Convergence Method (GCI method) given inCelik et al., (2008). This procedure is used to

241 estimate the numerical uncertainty and error due to discretization in CFD studies. The

242 methodology of this procedure is first to define a representative cell size. Second, select three

243 significantly different grid sets $N_{I}=90620$ cells, $N_{2}=158200$ cells, and $N_{3}=282050$ cells. The grids

244 are selected to make sure the refinement factors $r_{1}=h_{1} / h_{2}$ and $r_{2}=h_{2} / h_{3}$ are greater than 1.3,

245 where $h_{i}=\left[\frac{1}{N_{i}} \sum_{j=1}^{N_{i}}\left(\Delta A_{j}\right)\right]^{\frac{1}{2}}$ is the representative cell size for the $i$ th grid.The drag coefficient

246 value is determined as the key variable of each grid.The grid convergence index (GCI value) is

247 calculated to estimate discretization errors between grids. The GCI value for the finer grid should

248 be relatively small to show that the solution canbe replicated when the finest grid is employed. In

249 the present study, the GCI value between meshes $N_{l}$ and $N_{2}$ is $2.9 \%$ and the value between meshes

$250 N_{2}$ and $N_{3}$ is $1.1 \%$. This indicated that the mesh size used in the study, $N_{3}$, provides results that

251 can be considered mesh independent.The detailed description of the method is presented by

252 Celik et al., (2008).

253 A structured, hexahedral mesh was used for the computations. The mesh is finer closer to

254 the plate walls and downstream of the plateto resolve the boundary layer and to observe vortex 255 shedding.Fig. 5depicts the mesh in the vicinity of the plate. 


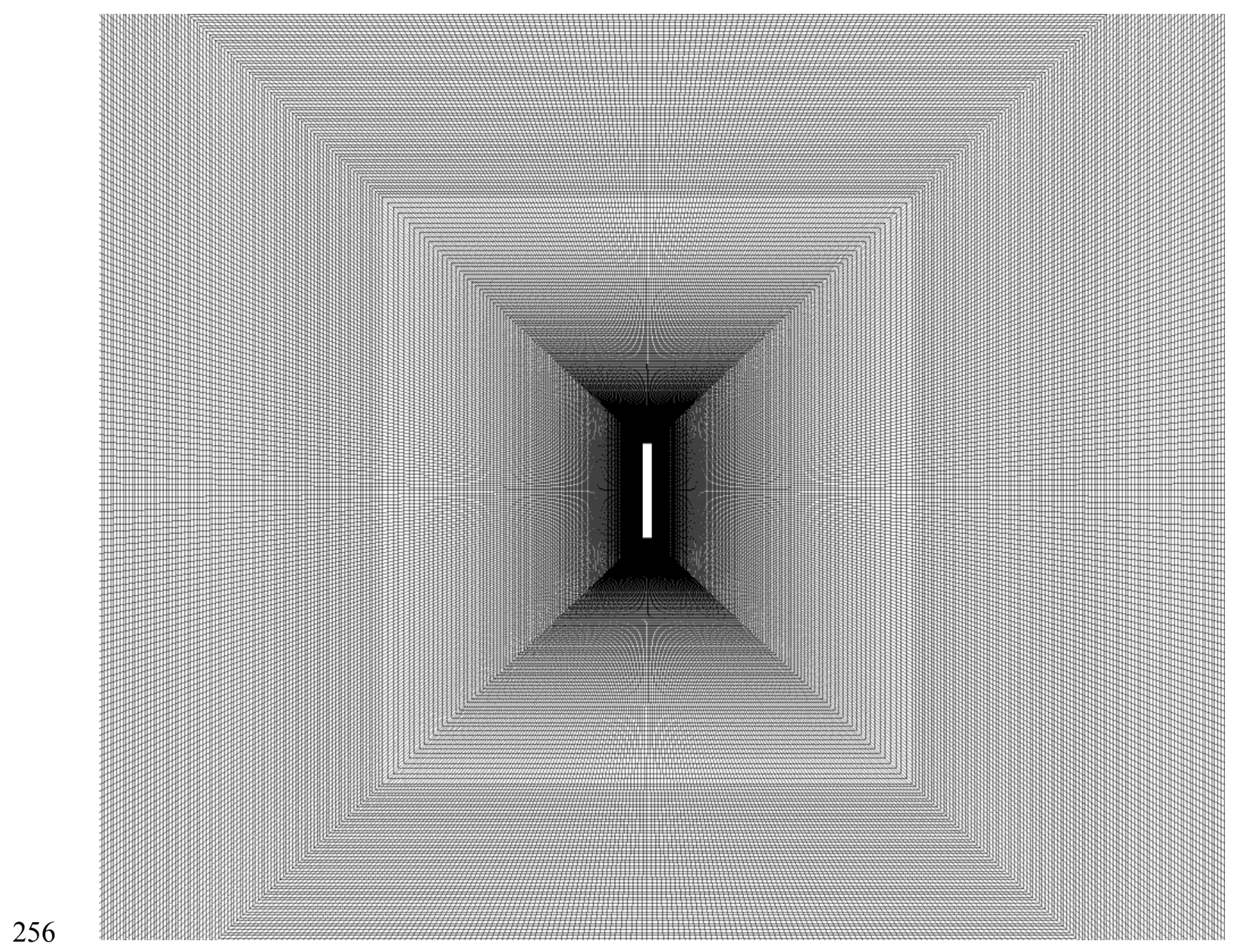

Fig.5. Structured mesh in the vicinity of the plate.

Discrete time steps are used in transient simulations. The sensitivity of the simulation

260 results to the time step size must be examined to accurately capture the unsteady flow field. A

261 temporal convergence test is conducted for the case of plate depth equal $0.6 \mathrm{~L}$. Drag coefficients

262 are determined for three time step sizes $\Delta t=0.008 \mathrm{~s}, 0.0016 \mathrm{~s}$, and $0.00032 \mathrm{~s}$ with corresponding

263 values of $3.32,3.55$, and 3.55 . This shows that $\Delta t=0.0016$ sis sufficient to ensure the temporal

264 convergence. 
A non-dimensional wall distance in a wall-bounded flow is defined as $y^{+}=u_{*} y / v$,

266 where $u_{*}$ is the friction velocity at the nearest wall andyis the distance to the nearest wall. The

267 average $y^{+}$value at the plate wall for cases of $F r=0.18,0.65,0.71,0.75$, and 0.92 are 4.76, 3.31,

$2682.81,2.57$, and 1.80, respectively. The $y^{+}$values are all below 5, ensuring that the viscous

269 sublayer is numerically resolved. The Courant number $(C=u \Delta t / \Delta x)$ is a non-dimensional

270 parameter that describes how fast flow information is propagated through the computational

271 domain. The Courant-Friedrichs-Lewy (CFL) condition is a numerical stability criterion that

272 requires $C \leq 1$ for a stable, unsteady solution. The averagevalue of the Courant number for all

273 plate depths cases are all below unity.The maximum value of thelocal Courant number is 46.2 ,

274 whichoccurs at a few cellsnear the top and the bottom plate corners.

\section{Results and Discussion}

For additional validation of the model,resultspredicted by the multiphase model are

277 compared tothose predicted by the single-phase model. The single-phase simulation considersthe

278 flow past a platein a nearlyinfinite fluid domain. In order to make the boundary conditions in

279 multiphase simulation similarto the single-phase simulation, the plateisfully submerged in the

280 channel and oriented at a distance of approximately $7.5 \mathrm{~L}$ away from the water surface. The

281 volume fraction contour of multiphase simulation isillustrated inFig. 6.As shown inFig. 6, the

282 water depth of the multiphase simulation is set to be the same as the channel height in the single-

283 phase simulation. Fig. 6showsno disturbances at the water surface, which suggests theflow field

284 near the plateis not influenced by the presence of the free surface. Therefore, the results for the 285 multiphase simulation should be identical with the single-phase simulation. 


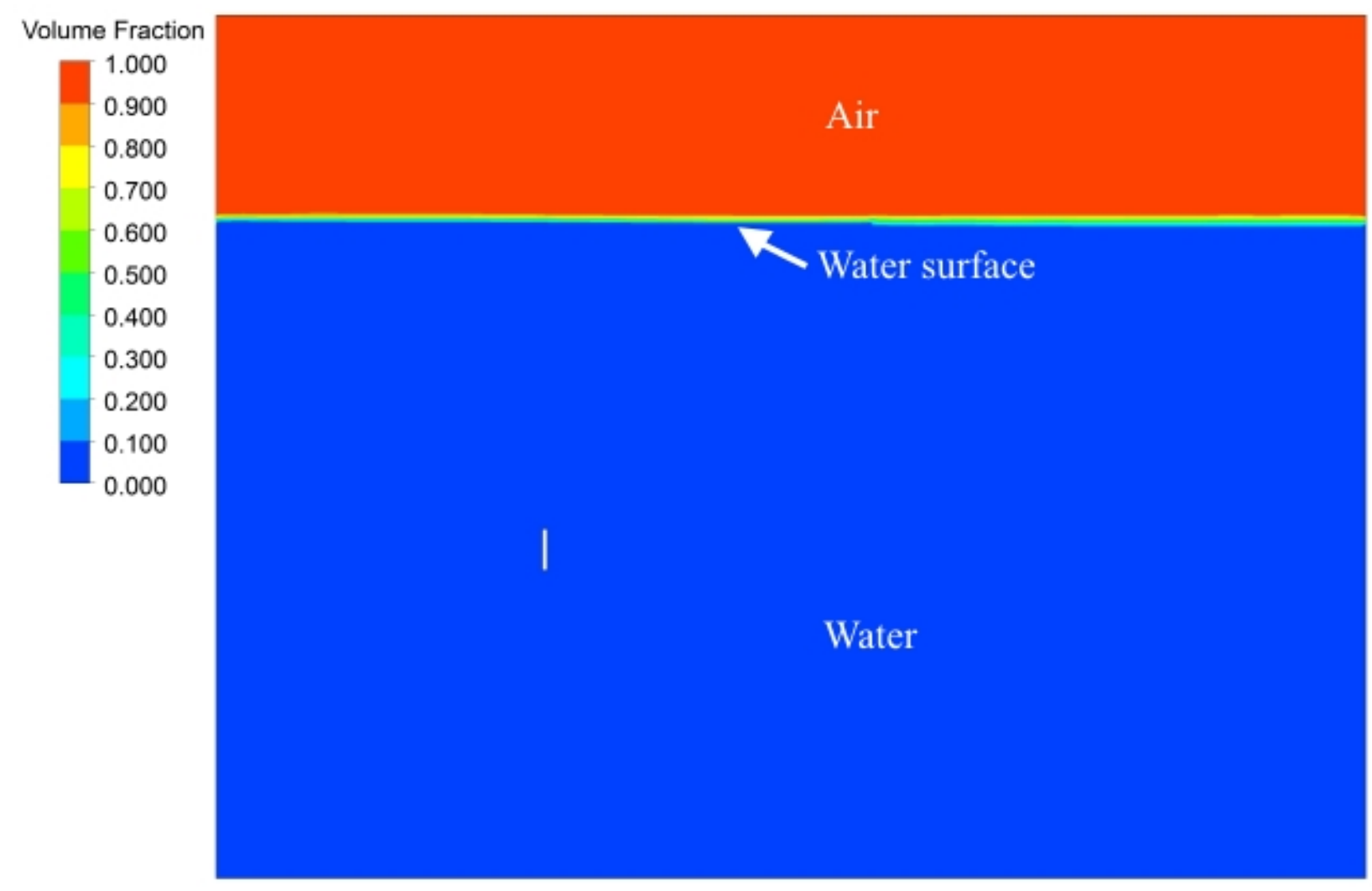

Fig.6 Volume fraction contour predicted by multiphase simulation.

Fig. 7depicts the same velocity and vorticity distribution for both single-phase and 290 multiphase simulations. Fig. 7(b)shows the vortices shed from both the top and bottom surfaces 291 of the plate interact with one another, which then gradually dissipate downstream. The vortices 292 areshed alternatively from the top and the bottom corner of the plate and generate a regular flow 293 pattern in the wake. Other than the volume fraction and the vorticity contour, the mean drag 294 coefficient and the Strouhal number are calculated from at least ten vortex shedding cycles. As 295 reported by many earlier investigators such as Tamura et al., (1990); Mittal and Balachandar, 296 (1995); Najjar and Vanka, (1995);Bosch and Rodi, (1998);Najjar and Balachandar, (1998); and 297 Tian et al., (2013), the two-dimensional flow simulation over predicts the drag 298 coefficients.Najjar and Balachandar, (1998)compared the numerical results for two-dimensional 
299 and three-dimensional simulations, indicating that the three-dimensional effect is the reason for 300 the over prediction of the two-dimensional simulations. The present study is in agreement with 301 the two-dimensional numerical results reported in the literature.The drag coefficient value and 302 the Strouhal number for the single-phase simulation are 3.86 and 0.125 , while the same values 303 predictedby the multi-phase simulation are 3.86 and 0.126 , respectively.Results show that the 304 single-phase and multiphase simulations yield identical values for desired performance 305 characteristics regardless of the use of multiphase VOF model when the plate is sufficiently far 306 away from the free surface. 
Velocity
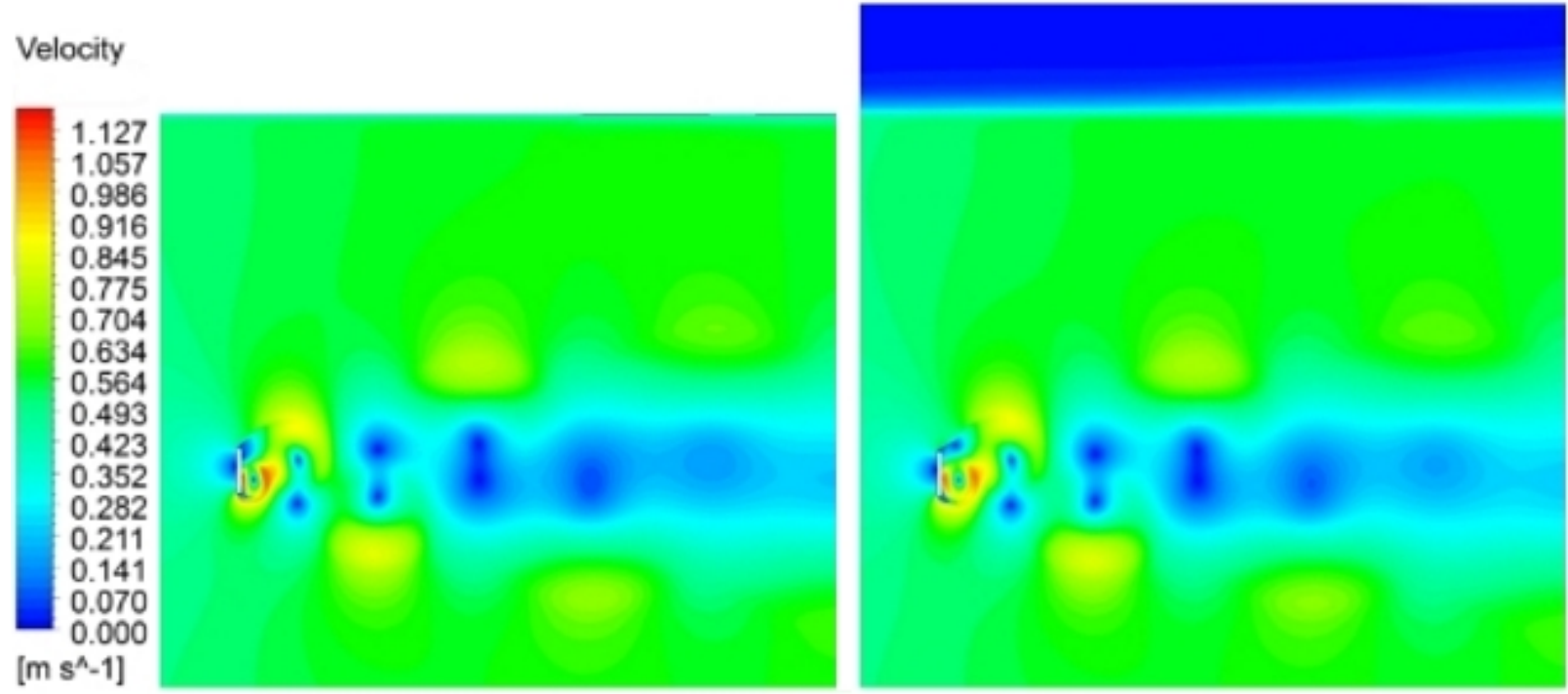

(a)
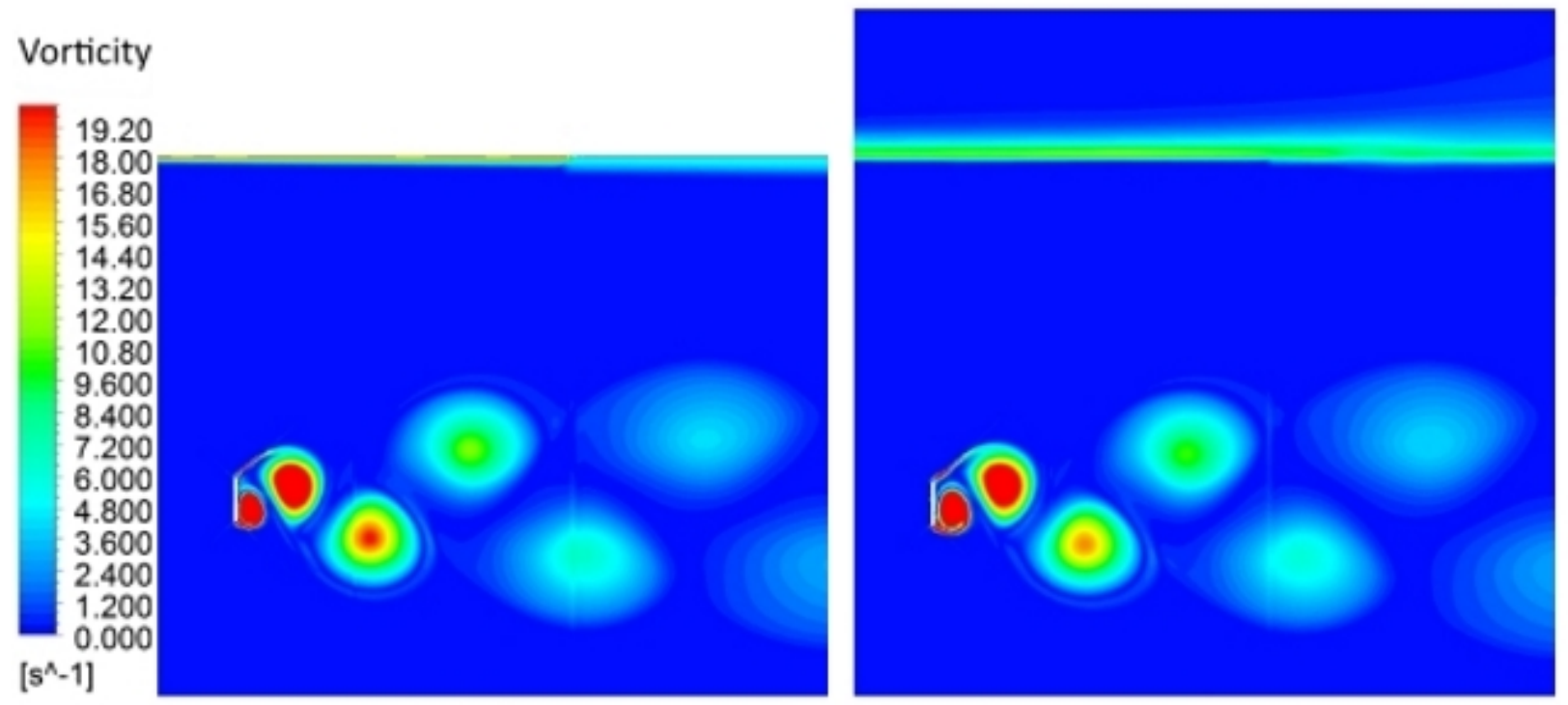

(b)

308 Fig.7. Instantaneous (a) velocity and (b) vorticity contours. Results of single-phase simulation is 309 shown in the left column and the results of multiphase simulationis shown in the right column.

Investigating the free surface effects was the primary focus of this manuscript.As

312 previously mentioned, the three-dimensional validation simulation was conducted to assure the

313 accuracy of the mathematical model and the numerical method; however, two-dimensional

314 simulations were conducted because three-dimensional simulations were too computationally 
315 expensive for the time allotted for this study.Itis observed that the proximity of the plate to the 316 surface has a profound effect on the spatial and temporal characteristics of the vorticity and 317 velocity field. The wake structure is distinctly different from the deeply submerged plate.

318 Fig. 8showsthe instantaneous volume fraction contour at different plate depths. The 319 volume fraction contour depictsthe local air and water fraction, where zero represents pure water 320 and one represents pure air. When the plateis submerged $7.5 L(F r=0.18)$, no surface deformation 321 is observed. When the plateis $0.6 \mathrm{Lsubmerged}(F r=0.65)$, some surface deformation is observed. 322 The flow above the plate interacts with the surface, resulted in a drop in the surface right behind 323 the plate. The surface wave decays in magnitude far downstream of the plate. When the 324 plateissubmerged $0.3 L(F r=0.92)$, additional fluctuation along the surface is observed. As 325 depicted in Fig. 8(c), more air is entrained into the water region behind the plate and results in 326 larger surface distortion. 


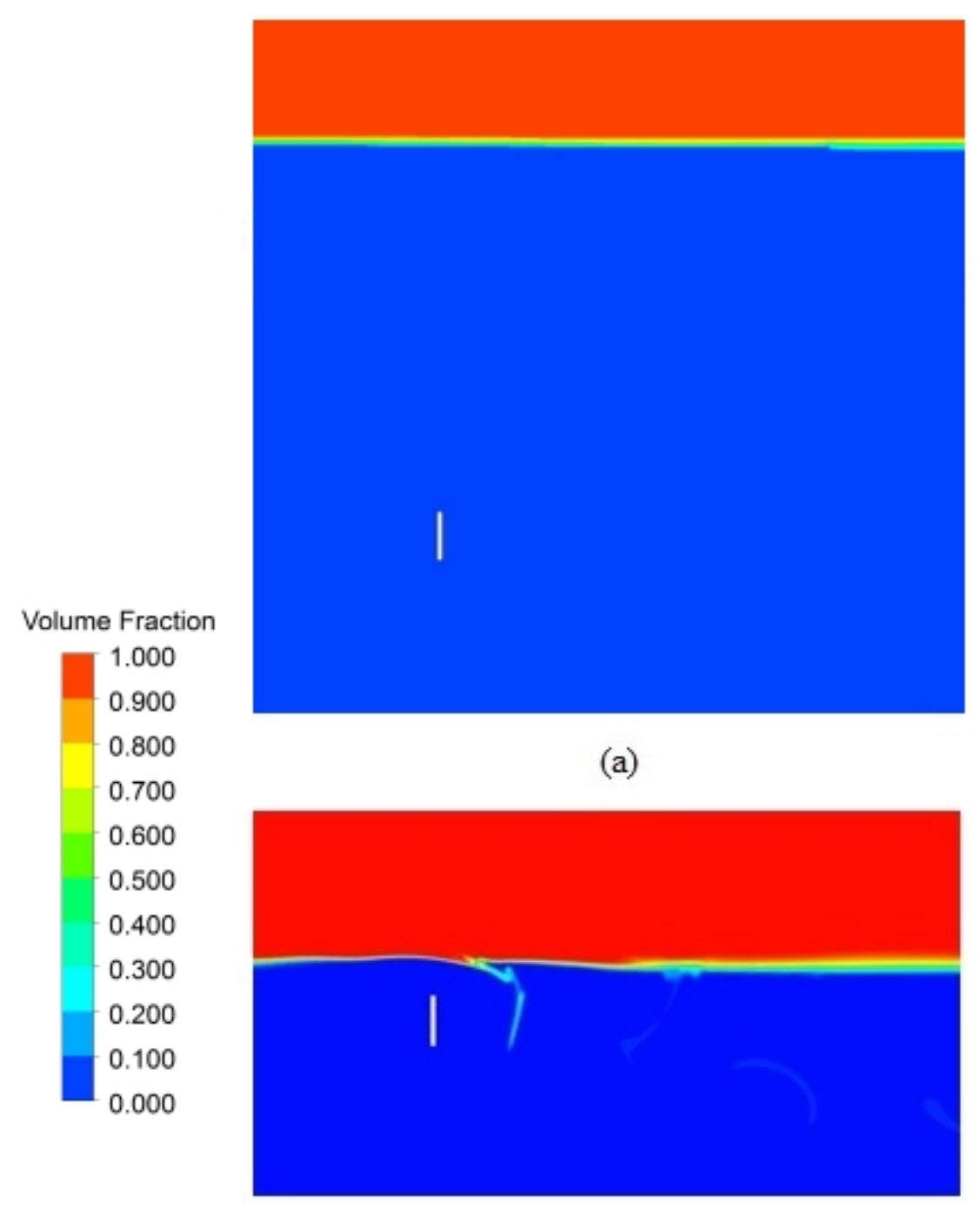

(b)

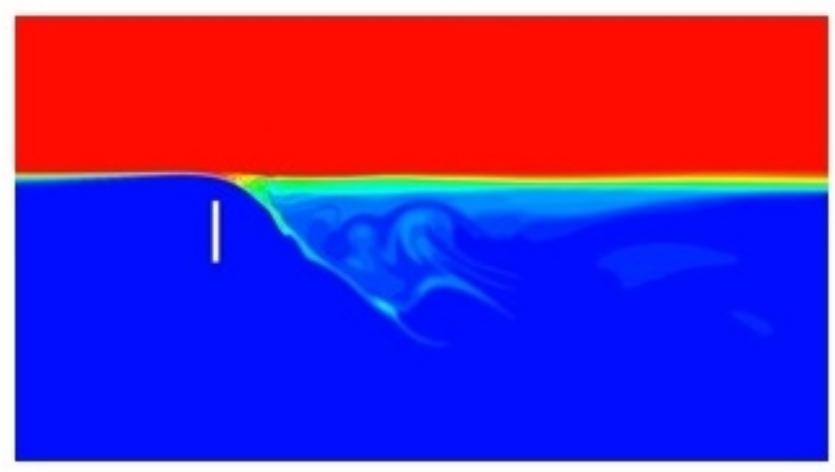

(c) 
The present study observes similar flow featuresas depicted in Miyata et al., (1990) and

331 Sheridan et al., (1997). Fig. 9illustratesthe velocity and vorticity contourfor different values of

332 the Froude number. The black horizontal line above the plate shown in Fig. 9 (b) and (c)

333 indicates the free surface location. The free surface penetrates into the plate's near wake as the

334 Froude number becomes larger. Vortex shedding alternating from upper and lower plate

335 boundary is observed in all cases. The vorticity contour showsthat the free surface hasa drastic

336 effect on the flow. The vortex shedding is symmetric and regular for $F r=0.18$ as seen inFig. 9

337 (a).For the cases of $F r=0.65$ and 0.92(Fig. 9 (b) and Fig. 9 (c)), the upper vortex shedding

338 componentis influenced by the free surface, resulting in asymmetric vortex shedding and

339 irregular flow pattern in the wake. As shown in Fig. 9 (b) for the case of $F r=0.65$, the large scale

340 vortex shedding is asymmetric. The upper vortices are dissipated by the free surface and then

341 breakinto small eddies near the surface, leaving the bottom vortices to dissipate further

342 downstream. When the plate approaches even closer to the surface as shown in Fig. 9 (c) for the

343 case of $F r=0.95$, a vortex formsabove the top of the plate and adjacent to the free surface. A jet-

344 like flow is observedfrom the free surface on top of the plate. This jet-like flow is dominated by

345 the vortices generated from the free surface, whichinduce strong free surface

346 deformation. Smaller vortices are seen in this case compared to others. The jet flow tends to

347 merge with the vortices originating from the bottom of the plate. The vortex shedding from top

348 and bottom of the prismare pushed downward due to the presence of the jet-like flow.Moreover,

349 the upper vorticesare not only compromised by the free surface, but are also blended together

350 with the bottom vortices and dissipate together downstream and the wake flow becomes even

351 more irregular.Characteristics of the drag and lift coefficients are directly affected by different

352 vortex shedding motions. 


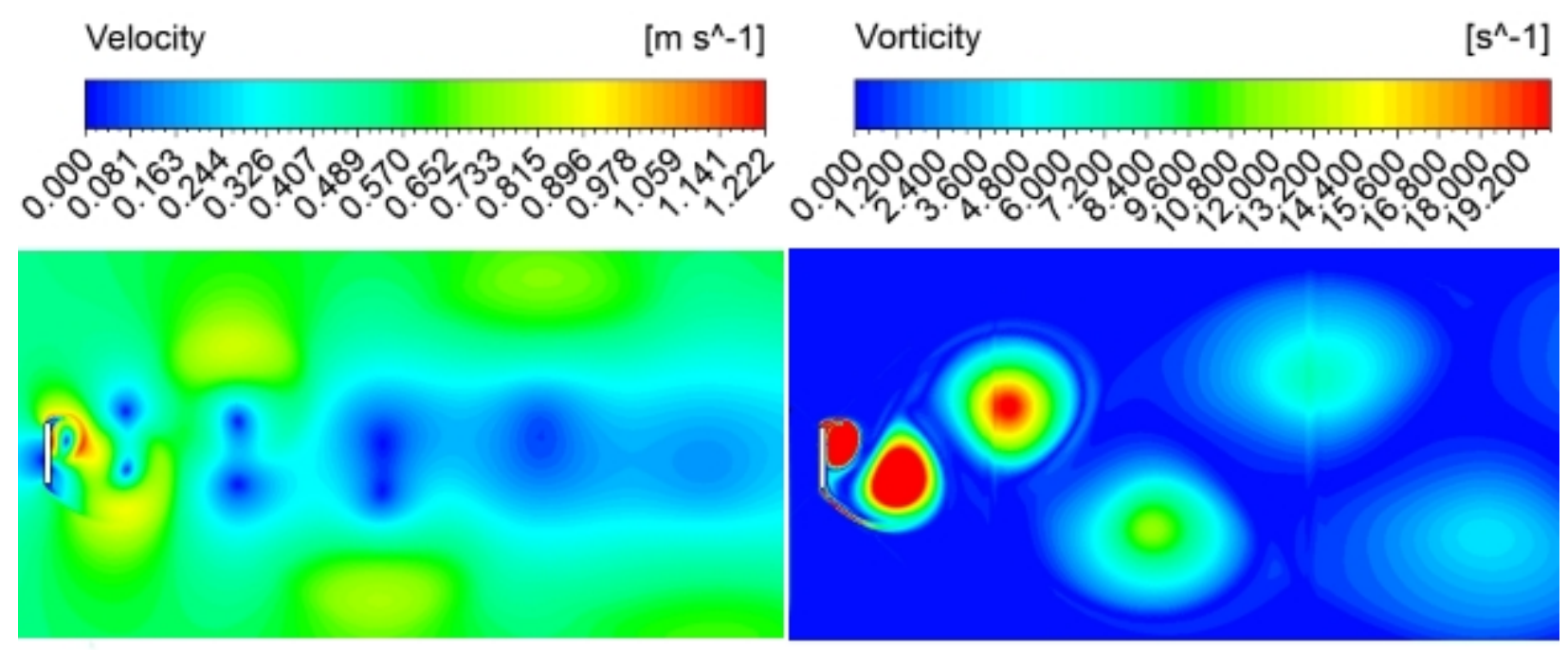

(a) $\mathrm{Fr}=0.18$

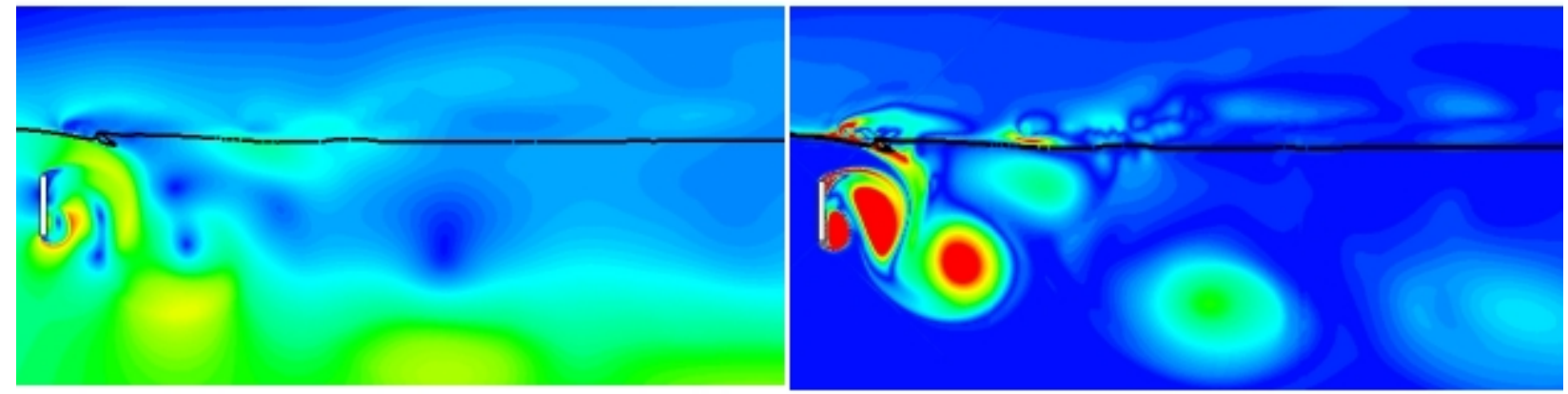

(b) $\mathrm{Fr}=0.65$

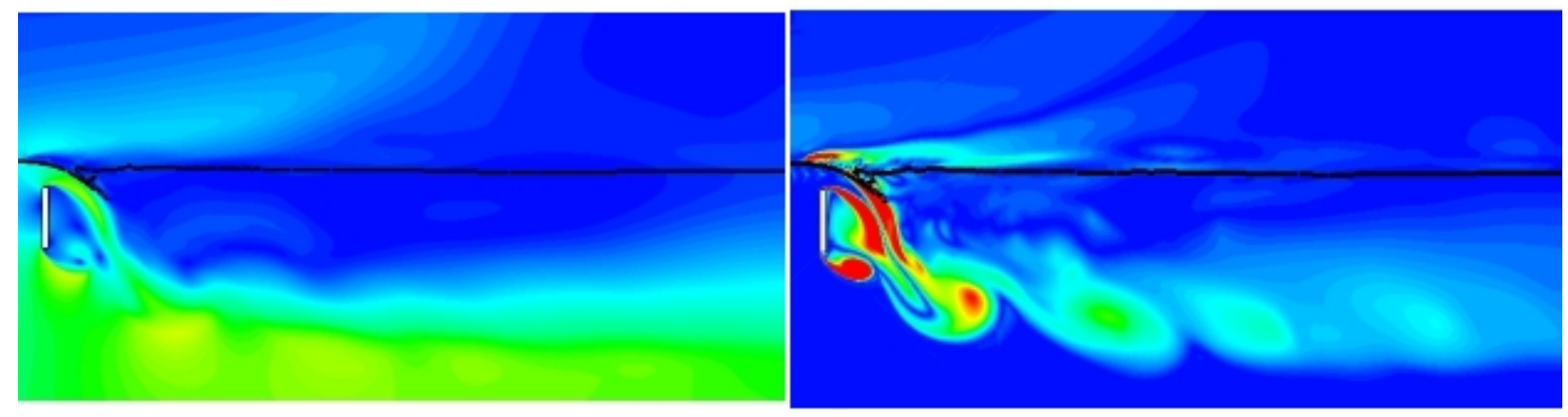

(c) $\mathrm{Fr}=0.97$

354 Fig.9.Instantaneous velocity and vorticity contoursfor (a) $F r=0.18$ at $\mathrm{t}=26.4 \mathrm{~s}$, (b) $F r=0.65$ at $\mathrm{t}=$ $355 \quad 28.8 \mathrm{~s}$ and (c) $F r=0.92$ at $\mathrm{t}=28 \mathrm{~s}$.

The profiles of time averagedstreamwise component of the velocity at the upstream and

358 the downstream of the plate are shown in Fig. 10for $F r=0.65$. The velocity profiles are averaged 
over5 vortex shedding cycles.Upstream velocity profiles are plotted at locations $2.5 L, 5 L, 12 L$,

$36014 L$, and $16 L$ away from the plate,as depicted in Fig. 10(a). The velocity profiles at about $12 L$

361 and $14 L$ from the plate are nearly the same; suggesting that the fully developed flow conditions

362 are attained at these locations. The velocity profiles approaching plates $(5 L$ and $2.5 L)$ are

363 influenced by the presence of the plate and the free surface. The water speed decreasesnear the

364 free surface due to the slower air velocity above. Downstream velocity profiles are plotted $1 L$,

$3652.5 L, 5 L$, and $10 L$ away from the plate, as depictedin Fig. 10(b). Irregular flow pattern caused by

366 the interaction of the vortices shed by the plate and the free surface effects in near wake velocity

367 profiles is shown at $1 L$ and $2.5 L$ away from the plate. Further away from the plate at $5 L$ and $10 L$

368 the velocity profiles gradually recover to regular wake flow patterns.
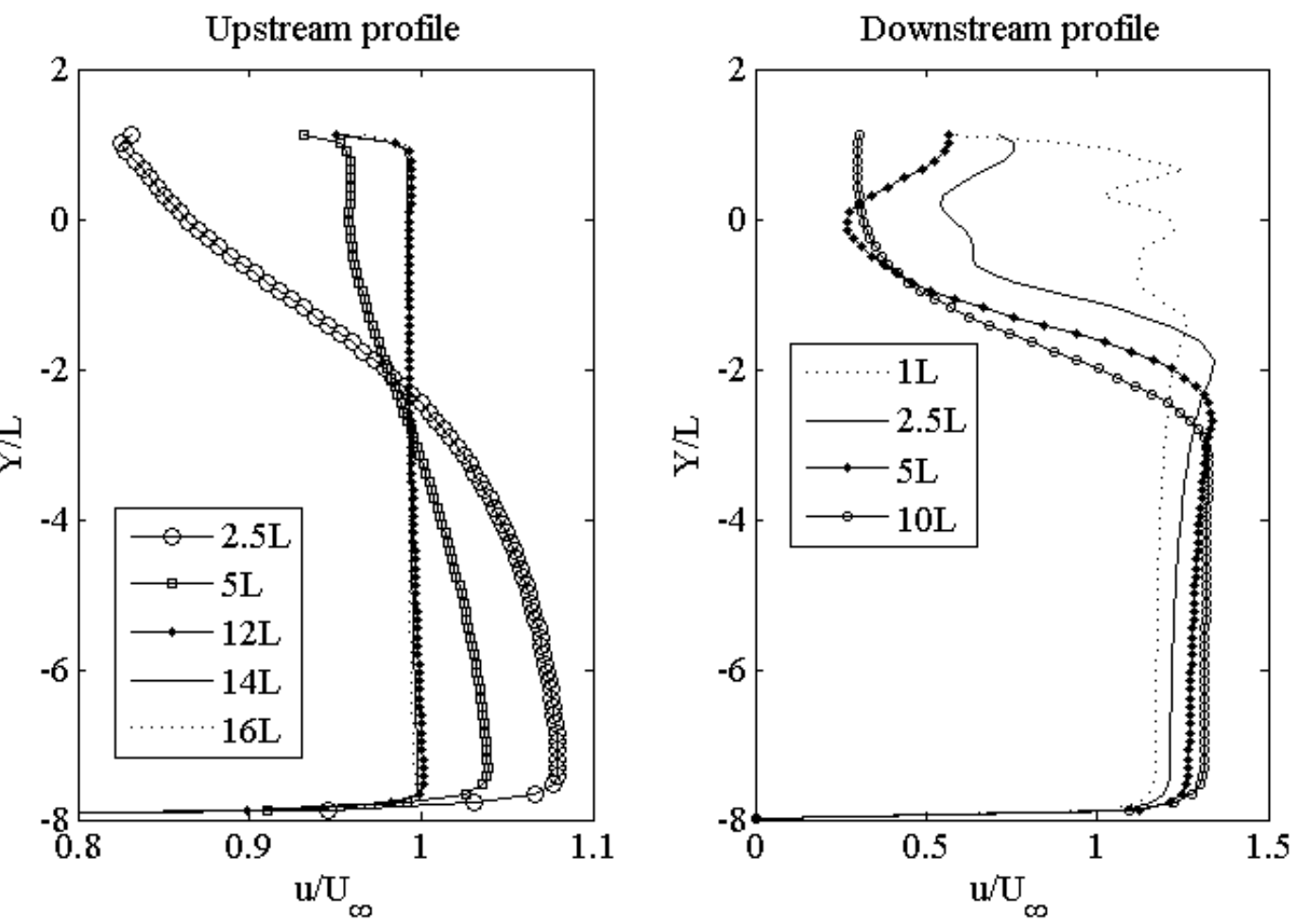

370 Fig.10. Normalized profiles of the time averaged streamwise component of velocity for $F r=$ 3710.65 at various locations (a) upstream and (b) downstream of the plate. 
Fig. 11shows the drag and the lift coefficient as functions of time for various values of

373 the Froude number. The mean values of the force coefficientsare calculated when the periodic

374 state is reached. Mean values are determined from the time signature of drag and the lift

375 coefficientat flow time between 30 sto 40 s which spans nearly 6 vortex shedding cycles. It is seen

376 that the drag coefficient $\left(C_{D}\right)$ has a mean value of 3.86 in the case of $F r=0.18$ (where the plateis

377 far away from the surface). In the case of $F r=0.65, C_{D}$ decreases to 3.55 . By further decreasing

378 the submergence depth to the case of $F r=0.92, C_{D}$ reduces to 2.18 , corresponding to a $38 \%$

379 droprelative to the case of $F r=0.65$. Results show that the influence of the free surface is

380 significantwhen the plateapproaches the free surface.The different vortex shedding motion

381 effectsis revealed when investigating the variation for both drag and lift coefficients. The drag

382 and the lift coefficients display periodic behavior for all cases. When the plateis far away from

383 the free surface, (seeFig. 11(a)), the vortex shedding in this case is symmetric and very regular.

384 When the plateis near the free surface, (seeFig. 11(b) and (c)), the drag and lift coefficients

385 havemore complicateddynamics with several modes present.InFig. 11(c), the variation of the

386 force coefficients showsthat multiple wave motionsare combineddue to the domination of the jet-

387 like flow formed from the free surface.Table 3lists the mean and the root mean square (rms) of

388 fluctuating force coefficients and the Strouhal number determined for various values of $F r$. The

389 drag coefficient decreases from $3.86(F r=0.18)$ to $3.55(F r=0.65)$ and then rapidly drops to

3902.75 at $F r=0.71$. The drag coefficient does not change as much as $F r$ increases from 0.71 to

3910.75 , whileit decreases substantially to $2.18 \mathrm{as} F r$ is increased to 0.92 . The rmsof $C_{D}$ fluctuation at

392 first increases to $0.51(F r=0.65)$ and then drops significantly to $0.29(F r=0.71)$. Another

393 significant drop from $0.27(F r=0.75)$ to $0.07(F r=0.92)$ is also obtained.Past studies of flow

394 past a circular cylinder havereported a reduction in drag coefficient and anincrease in the 
395 Strouhal number when reducing the gap ratio between the cylinder and the free surface. The 396 Strouhal number increasesfrom $0.162(F r=0.65)$ to $0.355(F r=0.92)$, and simultaneously the 397 rms of fluctuating lift force decreases. The change of vortex shedding frequency is closely related 398 to the substantial decrease of drag coefficient. Furthermore, the lift coefficient shows that there 399 are several modes of flow transitions dominating the flow. This impliesthat the vortex shedding 400 at shallow depthsis not governed by a single mode in comparison when the prismis submerged in 401 anearly infinite flow domain. 

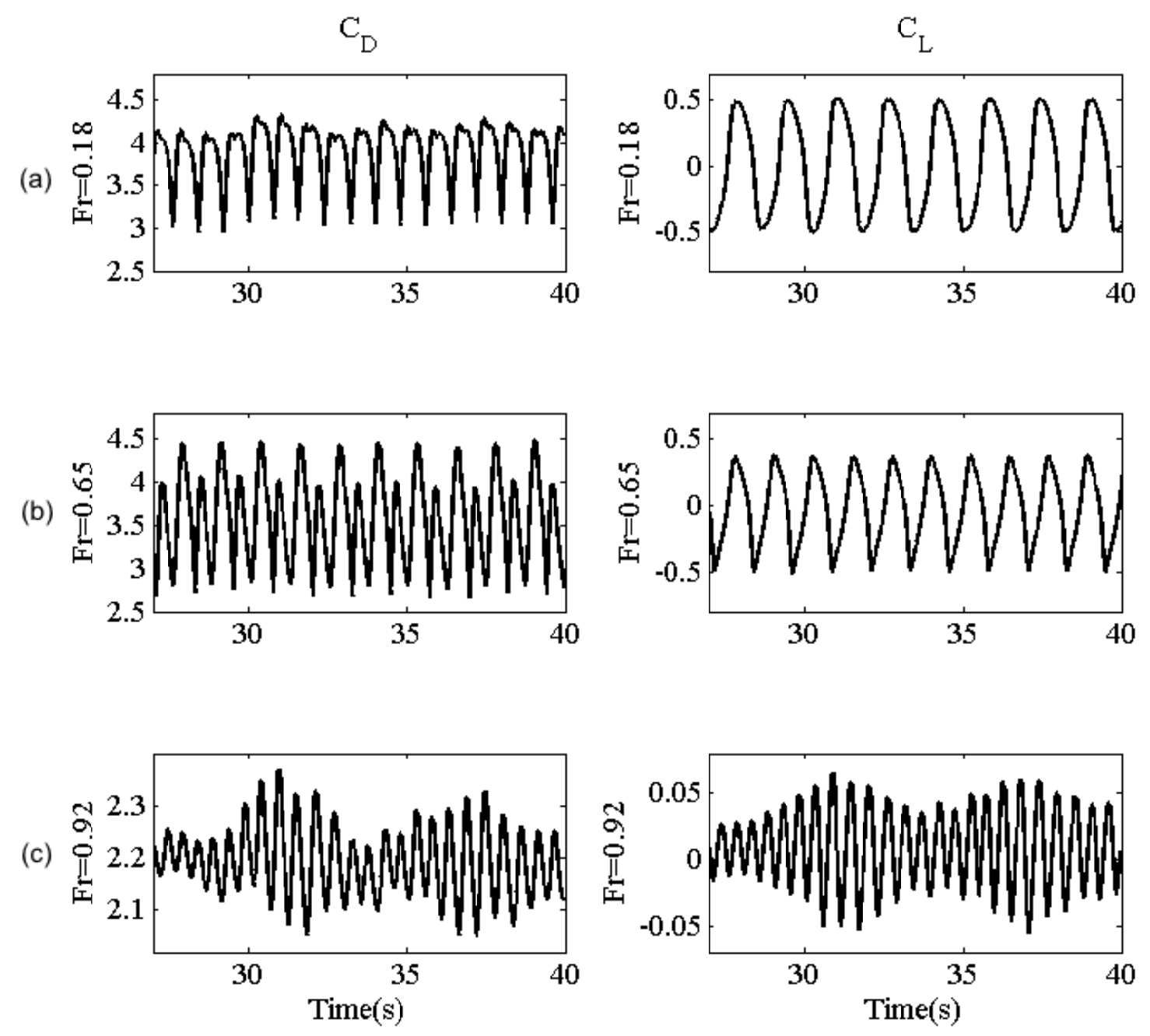

405

409 Fig.11. Drag and lift coefficients as functions of time for (a) $F r=0.18$ (b) $F r=0.65$ (c) $F r=$ $410 \quad 0.92$.

408

412 Table 3. Mean and root mean square of fluctuating drag and lift coefficients and the Strouhal 413 number for different values of theFroude number

\begin{tabular}{|lccccc|}
\hline & $\mathbf{F r}=\mathbf{0 . 1 8}$ & $\mathbf{F r}=\mathbf{0 . 6 5}$ & $\mathbf{F r}=\mathbf{0 . 7 1}$ & $\mathbf{F r}=\mathbf{0 . 7 5}$ & $\mathbf{F r}=\mathbf{0 . 9 2}$ \\
$\boldsymbol{C}_{\boldsymbol{D}}$ & 3.86 & 3.55 & 2.75 & 2.65 & 2.18 \\
$\boldsymbol{C}_{\text {Drms }}$ & 0.36 & 0.51 & 0.29 & 0.27 & 0.07 \\
$\boldsymbol{C}_{\boldsymbol{L}}$ & 0.00 & -0.02 & -0.04 & -0.03 & 0.01 \\
$\boldsymbol{C}_{\mathbf{L r m s}}$ & 0.38 & 0.28 & 0.17 & 0.14 & 0.03 \\
$\boldsymbol{S t}$ & 0.125 & 0.162 & 0.208 & 0.233 & 0.355 \\
\hline
\end{tabular}


The cumulative mean of drag and lift coefficientasa function of time is shown inFig.

40912 for various values of $F r$. The mean of force coefficients is calculated starting at $20 \mathrm{~s}$ by using

410 the instantaneous data at every $0.0016 \mathrm{~s}$. The cumulative mean values tend to constant

411 asymptotically, implying that the force coefficients have reached stability. The mean values of

412 the force coefficient listed in Table 3 have relative error less than $5 \%$ when compared to the

413 asymptotic values of the cumulative mean of force coefficients. This discrepancy is due to the

414 initial transient at early simulation time influences the overall cumulative mean values. As

415 simulations are carried out further in time this difference naturally becomes smaller.
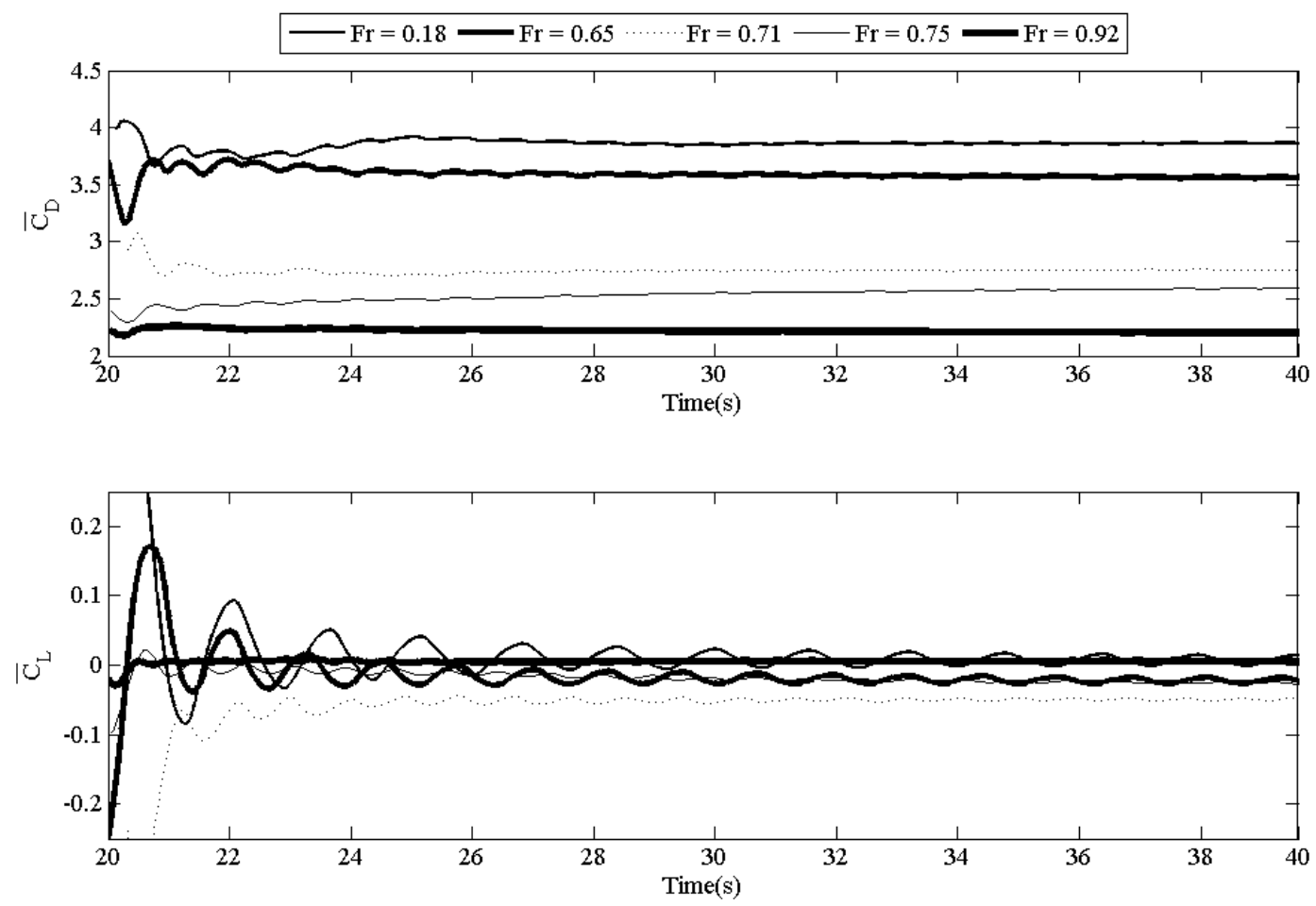

416

417 Fig.12. Cumulative mean of drag and lift coefficients for $F r=0.18,0.65,0.71,0.75$ and 0.92. 
420 a solid surface (no-slip surface) replacing thefree surface. The solid surface is placed $0.5 L$ away

421 from the top of the plate, this case would correspond to $F r=0.71$ if the free surface is present.

422 The mean value of the drag coefficient is calculated to be 3.67 with the solid surface, which is

423 much closer to the value of the drag coefficient, 3.86, for a plate in an infinite domain. As

424 presented above the drag coefficient is 2.75 for $F r=0.71$. It can be concluded that the reduction

425 in drag coefficient is not simply by the confinement. The interaction of the free surface waves and

426 the wake flow should be the reason for drag reduction. This does not rule out the possibility of

427 the adverse influence of the air entrainment deep into the wake, as seen in Fig. 8.

\section{6. Conclusions}

429 Numerical simulationswere performed to understand the flow structure about a 430 plateplaced near the free surface.The free surface effect on the platewas investigated by 431 performing simulations at $7.5 \mathrm{~L}, 0.6 \mathrm{~L}, 0.5 \mathrm{~L}, 0.45 \mathrm{~L}$, and $0.3 \mathrm{~L}$ depths corresponding to local Froude 432 number values of $0.18,0.65,0.71,0.75$, and 0.92 . Two-dimensional transient simulations 433 wereconducted for the Reynolds number of 50,000. The $k-\omega$ SST turbulence model and VOF 434 multiphase model were employed with a transient SIMPLE solver to solve flow past a plate close 435 to the free surface.A three-dimensional validation simulation was conducted to compare with a 436 previously published experimental result presented in Malavasi and Guadagnini, (2007). 437 Theresults for both drag and lift coefficients and the Strouhal number match well with their 438 results. Simulation accuracyis verified by performingspatial and temporal discretization 439 convergence tests, providing a GCI value of $1.1 \%$ on drag coefficient for a 282050 cell mesh and $440 \quad$ time step size of $\Delta t=0.0016 \mathrm{~s}$. 
The presented results showed that as the depth decreased from $0.6 L$ (i.e., $F r=0.65$ ) to

$4420.3 L$ (i.e., $F r=0.92$ ), thedrag coefficientdecreased $38 \%$ from 3.55 to 2.18.The Strouhal

443 numberincreased from 0.162 to 0.355 . The substantial changes observedare similar to flow past a

444 circular cylinder close to the free surface. The variation of drag coefficient with depth was

445 consistent with the results reported in Miyata et al., (1990). The flow pattern observed is also

446 consistent with the results in Sheridan et al., (1997) andReichl et al., (2005). The wake structure

447 for a near-surface plateis distinctly different from the wake of a deeply submerged plate. The

448 wake flowbecomesmore irregular as depth decreases. Thevortex formed from the top of the plate

449 break into small eddies due to the presence of the free surface. Furthermore, a jet-like flow

450 formed from the surface on top of the platewas observed, which weakened the upper vortices and

451 results in large surface deformation. The presented study demonstrates that the proximity of the

452 blades to the free surface has profound influence on the energyharvesting devices performance in

453 the river or ocean currents. The rapid decrease in drag coefficient occurs between a depth of $0.6 \mathrm{~L}$

$454(F r=0.65)$ and $0.5 L(F r=0.71)$ at the flow conditions considered, therefore, the plate depths

455 greater than $0.6 L$ are suggested for the energy harvesting devices utilizing rectangular prism 456 shape blades.

\section{Acknowledgment}

459 The authors would like to gratefully acknowledge funding from the Office of Naval Research for 460 this work under Award N00012-14-C-0078 through a SBIR grant with e-Harvest.

\section{References}


ANSYS FLUENT Theory Guide Release 14.5, 2011.

ANSYS FLUENT User Guide Release 14.5, 2011.

Arslan, T., Malavasi, S., Pettersen, B., Andersson, H.I., 2013. Turbulent Flow Around a SemiSubmerged Rectangular Cylinder. Journal of Offshore Mechanics and Arctic Engineering-Transactions of the Asme 135 (4).

Bayraktar, S., Yayla, S., Oztekin, A., Ma, H.L., 2014. Wall proximity effects on flow over cylinders with different cross sections. Canadian Journal of Physics 92 (10), 1141-1148.

Bosch, G., Rodi, W., 1998. Simulation of vortex shedding past a square cylinder with different turbulence models. International Journal for Numerical Methods in Fluids 28 (4), 601 616.

Celik, I., Ghia, U., Roache, P., Christopher, 2008. Procedure for estimation and reporting of uncertainty due to discretization in CFD applications. Journal of fluids EngineeringTransactions of the ASME 130 (7).

Fage, A., Johansen, F.C., 1927. On the Flow of Air behind an Inclined Flat Plate of Infinite Span. Proceedings of the Royal Society A: Mathematical, Physical and Engineering Sciences 116 (773), 170-197.

Hirt, C.W., Nichols, B.D., 1981. Volume of fluid (VOF) method for the dynamics of free boundaries. Journal of Computational Physics 39 (1), 201-225.

Knisely, C.W., 1990. Strouhal numbers of rectangular cylinders at incidence: A review and new data. Journal of Fluids and Structures 4 (4), 371-393.

Malavasi, S., Guadagnini, A., 2007. Interactions between a rectangular cylinder and a freesurface flow. Journal of Fluids and Structures 23 (8), 1137-1148.

Menter, F.R., 1993. Zonal two equation k- $\omega$ turbulence models for aerodynamic flows. AIAA 24th Fluid Dynamics Conference.

Menter, F.R., 1994. Two-equation eddy-viscosity turbulence models for engineering applications. AIAA Journal 32 (8), 1598-1605.

Mittal, R., Balachandar, S., 1995. Effect of three-dimensionality on the lift and drag of nominally two-dimensional cylinders. Physics of Fluids (1994) 7 (8), 1841-1865.

Miyata, H., Shikazono, N., Kanai, M., 1990. Forces on a circular cylinder advancing steadily beneath the free-surface. Ocean Engineering 17 (1-2), 81-104.

Monaghan, J.J., 1994. Simulating Free Surface Flows with SPH. Journal of Computational Physics 110 (2), 399-406.

Najjar, F.M., Balachandar, S., 1998. Low-frequency unsteadiness in the wake of a normal flat plate. Journal of Fluid Mechanics 370, 101-147.

Najjar, F.M., Vanka, S.P., 1995. Simulations of the Unsteady Separated Flow Past a Normal Flat-Plate. International Journal for Numerical Methods in Fluids 21 (7), 525-547.

Narasimhamurthy, V.D., Andersson, H.I., 2009. Numerical simulation of the turbulent wake behind a normal flat plate. International Journal of Heat and Fluid Flow 30 (6), 10371043. 
Negri, M., Cozzi, F., Malavasi, S., 2010. Self-synchronized phase averaging of PIV measurements in the base region of a rectangular cylinder. Meccanica 46 (2), 423-435.

Reichl, P., Hourigan, K., Thompson, M.C., 2005. Flow past a cylinder close to a free surface. Journal of Fluid Mechanics 533 (00), 269-296.

Schleicher, W., Ma, H., Riglin, J., Kraybill, Z., Wei, W., Klein, R., Oztekin, A., 2014a. Characteristics of a micro-hydro turbine. Journal of Renewable and Sustainable Energy 6 (1), 1-14.

Schleicher, W.C., Riglin, J.D., Kraybill, Z.A., Oztekin, A., 2013. Design and simulation of a micro hydrokinetic turbine, 1st Marine Energy Technology Symposium, Washington, D.C.

Schleicher, W.C., Riglin, J.D., Oztekin, A., 2014b. Numerical Characterization of a Preliminary Portable Micro-hydrokinetic Turbine Rotor Design. Renewable Energy.

Schleicher, W.C., Riglin, J.D., Oztekin, A., Klein, R.C., 2014c. Numerical Optimization of a Portible Hydrokinetic Turbine, 2nd Marine Energy Technology Symposium, Seattle, WA.

Sheridan, J., Lin, J.C., Rockwell, D., 1997. Flow past a cylinder close to a free surface. Journal of Fluid Mechanics 330, 1-30.

Tamura, T., Ohta, I., Kuwahara, K., 1990. On the reliability of two-dimensional simulation for unsteady flows around a cylinder-type structure. Journal of Wind Engineering and Industrial Aerodynamics 35 (0), 275-298.

Tian, X., Ong, M.C., Yang, J., Myrhaug, D., 2013. Unsteady RANS simulations of flow around rectangular cylinders with different aspect ratios. Ocean Engineering 58 (0), 208-216.

Wilcox, D.C., 2006. Turbulence modeling for CFD, 3rd ed. DCW Industries, La Cãnada, Calif. 